\title{
Photographic observations of visual double stars $^{\star}$
}

\author{
D.M.D. Jasinta, M. Raharto, and E. Soegiartini
}

Bosscha Observatory, Lembang 40391, Bandung, Indonesia

Department of Astronomy, Institut Teknologi Bandung, Jl. Ganesha 10, Bandung 40132, Indonesia

Received May 25; accepted July 17, 1998

\begin{abstract}
The photographic observations of 72 visual double stars are presented. The observations were made in the years 1992-1997 with the 60-cm double refractor of the Bosscha Observatory at Lembang.
\end{abstract}

Key words: astrometry — binaries: visual

This is the continuation of the work published earlier (Jasinta et al. 1995). We present the photographic observations of visual double stars collected at the Bosscha Observatory. The table contains the analysis results for 72 pairs observed in the years 1992-1997, photographed in 90 plates.

The same method of observing, measuring, and reducing described by van Albada-van Dien (1983) was used. Plates with multiple exposures were taken with the $60-\mathrm{cm}$ Zeiss double-refractor. Trails were taken to determine the direction of the zero-point of the position angle. Plates were collected by Moedji Raharto. Measurements using the Leitz UWM3 machine were carried out by E. Soegiartini and D.M.D. Jasinta. Only good quality plates were measured.

A new computer programme developed by the first author was used for the reductions. Plate scale of $199^{\prime \prime} 169 \pm$ 0.'001 mm was adopted. Following the method of reduction by van Albada-van Dien (1983), we take the mean of different plates by taking plate error into account, by adding 120 to the square of the mean error of $\rho$ expressed in unit of 0 ". 001 . Unit weight was given to a resulting error of 0.1 . If there are more than one plate of the same object observed in the same year, the total weight is the sum of each weight.

Send offprint requests to: D.M.D. Jasinta

* Table 1 is only available in electronic form at the CDS via anonymous ftp to cdsarc.u-strasbg.fr (130.79.128.5) or via http://cdsweb.u-strasbg.fr/Abstract.html

\section{The table}

Column 1: CCDM number taken from Catalogue of the Components of Double and Multiple Stars (Dommanget \& Nys 1994).

Columns 2,3: Star name and component.

Columns 4,5: Magnitude and spectral type.

Column 6: Mean epoch of observations.

Column \%: Mean value of $\Delta \alpha \cos \delta$ in seconds of arc.

Column 8: Standard error of $\Delta \alpha \cos \delta$ in $0{ }^{\prime \prime} 001$.

Column 9: Mean value of $\Delta \delta$.

Column 10: Standard error of $\Delta \delta$ in $00^{\prime \prime} 001$.

Column 11: Position angle.

Column 12: Standard error of position angle in 0.01 .

Column 13: Separation.

Column 14: Standard error of separation in 0".001.

Columns 15-1\%: Number of plates, number of images, and sum of weight.

Columns 18: HIC numbers, as they appear in the CCDM. Two numbers indicate that the primary has a different number than the secondary.

Acknowledgements. We wish to acknowledge the Director of Leids Kerkhoven-Bosscha Fonds (LKBF) whose donations made this work possible. We appreciate as well Professor Bambang Hidayat for constant advice and encouragement.

\section{References}

Dommanget J., Nys O., 1994, R. Obs. Bel., Commun. Ser. A, No. 115

Jasinta D.M.D., Raharto M., Soegiartini E., 1995, A\&AS 114, 487

van Albada-van Dien E., 1983, A\&AS 52, 193 